\title{
Entanglement entropy of a superflow
}

\author{
Sergei Khlebnikov and Akhil Sheoran \\ Department of Physics and Astronomy, Purdue University, \\ West Lafayette, IN 47907, U.S.A. \\ E-mail: skhleb@purdue.edu, asheora@purdue.edu
}

AbSTRACT: We consider the theory of $N$ free Dirac fermions with a uniformly winding mass, $m e^{i q x}$, in two spacetime dimensions. This theory (which describes for instance a superconducting current in an $N$-channel wire) has been proposed to have a higher-spin gravity with scalar matter as the large- $N$ dual. To order $m^{2}$, however, thermodynamic quantities in it can be computed using standard general relativity instead. Here, we consider the question if the same is true for the entanglement entropy (EE). By comparing results obtained on two sides of the duality, we find that general relativity indeed accounts correctly for the EE of an interval to order $m^{2}$ (and all orders in $q$ ).

Keywords: AdS-CFT Correspondence, Holography and Condensed Matter Physics $(\mathrm{AdS} / \mathrm{CMT})$

ArXiv EPrint: 2111.03593 


\section{Contents}

1 Introduction 1

2 Asymptotic analysis 2

3 Thermal entropy $\quad 5$

$\begin{array}{lll}4 & \text { Entanglement entropy } & 7\end{array}$

5 Limit of a short interval $\quad 10$

6 Discussion 12

\section{Introduction}

In semiclassical gravity, the Gibbons-Hawking (GH) formula [1] interprets the Euclidean effective action of a spacetime in terms of free energy (of gravity plus matter) and thus provides a method for computation of gravitational entropy. If the spacetime is asymptotically anti-de Sitter (AdS), the same effective action also determines the entropy of the dual conformal field theory (CFT) in the context of AdS/CFT duality [2]. Furthermore, if the CFT is deformed by a relevant operator, the correspondence [3, 4] between operators in the CFT and fields in AdS tells us the precise way in which the entropy of the CFT can be computed from the action of a deformed spacetime.

Replacing the thermal density matrix in the von Neumann entropy formula with one obtained by integrating out a subset of degrees of freedom defines entanglement entropy (EE). It has been shown [5] that the EE of a spherical region in a CFT vacuum is the same as the entropy of a thermal state in an auxiliary hyperbolic space and that application of the AdS/CFT correspondence to this thermal state, in the case when the gravitational dual is standard general relativity, reproduces the result of the minimal-surface formula of Ryu and Takayanagi [6]. Furthermore, it has been argued [7], in a similar context, that the EE can be related to the area of a minimal surface directly, without going through a thermal state as an intermediary; that argument applies to a region of any shape.

In studies of the AdS/CFT correspondence, of special interest are cases when the CFT is solvable, e.g., a free theory. One such case is the theory of $N$ free Dirac fermions in two spacetime dimensions $(d=2)$, which has been conjectured [8] to be dual, in the large $N$ limit, to a higher-spin gravity with scalar matter in $\mathrm{AdS}_{3}$ [9]. Deformation of the CFT by a mass term corresponds to a nontrivial profile of a scalar. A scalar of amplitude $A$ sources the higher-spin fields at the same (quadratic [9]) order in $A$ as it sources the metric correction. To this order, however, neither those fields nor the metric correction contribute to variation of the action; the latter is given simply by the action of the scalar on the 
undeformed background. As a result, the $O\left(A^{2}\right)$ correction to the thermal entropy can be computed from the GH formula as if the dual theory were standard general relativity.

One may then wonder if to order $A^{2}$ the generalized GH formula [7] also works the same way as in general relativity, in particular, if the Ryu-Takayanagi (RT) formula for the EE still applies. In this paper, we address this question by making comparisons between results obtained on two sides of the duality.

The theory of $N$ Dirac fermions in $d=2$ is of interest and perhaps some practical importance in its own right. It can be used to describe a quantum wire with $N$ transverse channels. If we identify the chiral charge of the fermion with the electric charge in the wire, the fermion mass corresponds to a superconducting pairing amplitude induced for instance by proximity to a larger superconductor. The mass winding along the wire as $\tilde{m}(x)=m e^{i q x}$, where $m>0$ and $q$ are real constants, corresponds to a state with non-zero supercurrent. According to the preceding, we may hope to use standard general relativity to compute both the thermal and entanglement entropies to order $m^{2}$ and all orders in $q$.

Our main focus will be a non-vacuum, thermal state in the CFT, related holographically to the BTZ black hole [10]. In this case, there is potentially a small parameter $m / T$, where $T$ is the temperature. On the gravity side, a small $m / T$ corresponds to a small deformation of the BTZ background. In contrast, if $m$ is larger than both $T$ and $|q|$, the deformation cannot be small everywhere, and the only small dimensionless parameter proportional to $m$ is $m l$, where $l$ is the length of the entangling region. We briefly discuss this case towards the end of the paper.

We start in section 2 with establishing a relation between the scalar amplitude $A$ in gravity and the fermion mass $m$ in the CFT. The relation uses only the leading nearboundary asymptotics of the scalar and amounts to a computation of the scalar two-point function in the special, logarithmic case of the AdS/CFT correspondence. In section 3, we consider the thermal entropy and in section 4 the EE of an interval of length $l$ in a thermal state of the CFT. In both cases, we find agreement between $O\left(\mathrm{~m}^{2}\right)$ results obtained on two sides of the duality. For the EE, our evidence in mostly numerical, but in the case of a short interval, which we consider in section 5 , some results can be extracted analytically. In particular, the coefficient of the leading $(m l)^{2} \ln ^{2} l$ term in the $O\left(m^{2}\right)$ correction obtained from the RT formula is found to coincide with that obtained in ref. [11] directly in field theory. We present a discussion of our results in section 6 .

\section{Asymptotic analysis}

We consider deformations of a Euclidean non-rotating BTZ black hole [10], due to a smallamplitude complex scalar field $\phi$. The metric is of the form

$$
d s^{2}=r^{2} d \tau^{2}+F(r) d x^{2}+G(r) d r^{2}
$$

The asymptotic (near-boundary) region corresponds to large $r$, where $F(r) \rightarrow r^{2}$ and $G(r) \rightarrow 1 / r^{2}$, after we set the AdS radius to unity. The coordinates $x$ and $\tau$ are subject to 
the following periodicities:

$$
\begin{aligned}
& x \sim x+L_{x}, \\
& \tau \sim \tau+\beta .
\end{aligned}
$$

The GH formula interprets $T=1 / \beta$ as the temperature of the spacetime. According to the AdS/CFT correspondence [2], it is also the temperature in the dual CFT, while $L_{x}$ is the length of the spatial circle on which the CFT lives.

For the undeformed black hole

$$
F(r)=F_{0}(r) \equiv r^{2}+\alpha^{2}, \quad G(r)=G_{0}(r) \equiv\left[F_{0}(r)\right]^{-1}
$$

with

$$
\alpha=2 \pi T .
$$

The entropy of this space is $S_{0}=\alpha L_{x} /\left(4 G_{N}\right)$ [10], where $G_{N}$ is Newton's constant.

If we only want the Euclidean action of the deformed spacetime to order $A^{2}$ in the scalar amplitude $A$, we do not need to consider changes to the gravitational part of the action, since that part was extremal for $A=0$. This leaves us with the bilinear action of the scalar,

$$
I_{E}=\int d^{3} x \sqrt{g}\left(g^{m n} \partial_{m} \phi^{*} \partial_{n} \phi+M^{2} \phi^{*} \phi\right)
$$

where $g$ refers to the metric of the undeformed background. On the equations of motion, this reduces to the boundary term

$$
I_{E}=\left.\int d \tau d x\left(r g^{r r} \phi^{*} \partial_{r} \phi\right)\right|_{r=r_{m}},
$$

where $r_{m}$ is some large value of the radius.

Here, we focus on the logarithmic case $M^{2}=-1$, corresponding, via the standard dictionary $[3,4]$, to an operator of dimension $\Delta=1$ in the CFT. We restrict attention to static $x$-dependent solutions of the form

$$
\phi(x, r)=e^{i q x} \phi_{\mathrm{rad}}(r),
$$

with a constant momentum $q$ in the $x$ direction. The leading and first subleading terms in the asymptotic of the radial part $\phi_{\text {rad }}$ in this case can be combined into

$$
\phi_{\mathrm{rad}}(r)=\frac{A}{r} \ln (r \xi)+\ldots,
$$

where $A$ and $\xi$ are constants, and the dots stand for terms suppressed by inverse powers of $r$. The constant $\xi$ is a function of $q$ and $T$,

$$
\xi=\xi(q, T)
$$

but, in the present case, not of $A$ itself. For $q=0, \xi$ can be considered as a holographic definition of the correlation length. We will occasionally use this terminology also for $q \neq 0$. 
For a scalar described by a bilinear action on the undeformed BTZ background, the solution to the equations of motion is readily available, and one can read off it the full dependence of $\xi$ on its arguments. We will make use of this solution in the next section. Here, let us observe that both eqs. (2.7) and (2.9) depend only on the asymptotic form of the metric and so are a bit more general than the case at hand. For instance, the relation of $A$ to the mass parameter in the CFT, obtained using these equations, will apply also in the case of vacuum AdS, which has the same large- $r$ asymptotic as the BTZ metric (2.4) but a different topology.

Substituting eq. (2.9) in (2.7), we find that the action is divergent in the limit $r_{m} \rightarrow \infty$ and so needs to be renormalized. This is achieved by adding a boundary counterterm [12]. We do that by first stepping away from the logarithmic case, i.e., considering $M^{2}$ somewhat above -1 , so that

$$
\lambda \equiv 1+\left(1+M^{2}\right)^{1 / 2}=1+s
$$

with $0<s<1$, and then taking the limit $s \rightarrow 0$. The counterpart of eq. (2.9) is

$$
\phi_{\mathrm{rad}}(r)=A_{s} r^{s-1}\left[1-(\xi r)^{-2 s}\right]+\ldots,
$$

and the renormalized action, computed by the method of ref. [12], is

$$
I_{E, r e n}=2 s \int d \tau d x\left|A_{s}\right|^{2} \xi^{-2 s} .
$$

For eq. (2.12) to reproduce (2.9) in the limit $s \rightarrow 0, A_{s}$ must go infinity as $A /(2 s)$ with a constant $A$. We then see that the logarithmic case requires an additional subtraction, of the $O(1 / s)$ term in (2.13). After this subtraction, the renormalized action for $s=0$ becomes

$$
I_{E, r e n}=-\int d \tau d x|A|^{2} \ln (\mu \xi),
$$

where $\mu$ is a normalization momentum.

By the standard AdS/CFT dictionary [3, 4], the action (2.14) generates the two-point function of the corresponding operator in the dual CFT. In cases when this function can also be computed directly in field theory, we can, by comparing the two results, relate the scalar amplitude $A$ to the parameter accompanying the dual operator in the CFT Lagrangian. Consider, for the purposes of this comparison, the field theory of a $N$-component Dirac fermion in $d=2$. The operator in question there is $O=\bar{\Psi}_{R} \Psi_{L}$, where $\Psi_{L, R}$ are the left- and right-moving components of the fermion field. The source of $O$ in this theory is the position-dependent fermion mass $m e^{i q x}$, where we can choose $m$ to be real and positive. The two-point function, computed from a one-loop diagram is, to logarithmic accuracy, $(N / 2 \pi) \ln (\mu / Q)$ where $Q$ is the largest of the three mass scales: $m,|q|$, and $T$. Comparing this to eq. (2.14), we see that, if the results on two sides of the duality are to match, the amplitude $A$ in the gravitational theory must be related to $m$ as follows:

$$
A=m \sqrt{\frac{N}{2 \pi}},
$$

up to an inessential constant phase factor. 
We conclude this section with a comment concerning the relative magnitude of $q$ and $m$. In a superconductor, $m$ represents the energy gap and $q$ the superflow momentum per Cooper pair. It is well known that, in a conventional intrinsic superconductor, the $q / m$ ratio cannot be arbitrarily large: at a sufficiently large $q$, the gap goes to zero signaling transition to the normal state [13, section 4.4]. Here, however, we consider $q$ and $m$ as independent parameters, as may be the case when superconductivity is induced by the proximity effect. Accordingly, we extend our analysis to arbitrarily large $q$ (and indeed will not detect any obstacles to doing so).

\section{Thermal entropy}

According to the GH formula, the renormalized action (2.14) with the $\tau$ integral removed is interpreted as a correction $\delta \Omega_{\text {grav }}$ to the free energy of the black hole deformed by the classical scalar. Expressing the scalar amplitude $A$ through the fermion mass $m$ by eq. (2.15), we obtain

$$
\delta \Omega_{\text {grav }}(q, T)=-\frac{m^{2} N L_{x}}{2 \pi} \ln [\mu \xi(q, T)] .
$$

As already mentioned, for a scalar described by the bilinear action (2.6) on the undeformed BTZ background, the solution of the form (2.8) and the associated correlation length $\xi(q, T)$ can be readily found. Gravitational entropy obtained from eq. (3.1) can then be compared to the thermal entropy computed directly in the fermionic CFT deformed by the mass term. The computation of the gravitational entropy here is parallel to that done in ref. [7] for a scalar independent of $x$ but with an exponential dependence on the Euclidean time $\tau$.

The solution for the radial part of the scalar on the rigid BTZ background (2.4) is

$$
\phi_{\mathrm{rad}}(r)=\widetilde{A}(q, T)\left(r^{2}+\alpha^{2}\right)^{-\lambda / 2}{ }_{2} F_{1}\left(a, b, 1 ; \frac{r^{2}}{r^{2}+\alpha^{2}}\right)
$$

where the amplitude $\widetilde{A}$ depends, as we will see, on $q$ and $T ; \lambda$ is related to the mass squared of the scalar by eq. (2.11), and ${ }_{2} F_{1}$ is the hypergeometric function with

$$
a=\frac{1}{2}\left(\lambda+\frac{i q}{\alpha}\right), \quad b=\frac{1}{2}\left(\lambda-\frac{i q}{\alpha}\right) .
$$

As before, we focus on the case $\lambda=1\left(M^{2}=-1\right)$, when the asymptotic of ${ }_{2} F_{1}$ is logarith$\operatorname{mic}[14$, eq. (15.3.10)]:

$$
F(a, b, a+b ; z)=\frac{\Gamma(a+b)}{\Gamma(a) \Gamma(b)}[2 \psi(1)-\psi(a)-\psi(b)-\ln (1-z)]+O[(1-z) \ln (1-z)],
$$

where $\Gamma(\cdot)$ and $\psi(\cdot)$ are the gamma and digamma functions, respectively. The asymptotic form of the solution then agrees with eq. (2.9), with

$$
\begin{aligned}
A & =\frac{2 \widetilde{A}(q, T)}{\pi} \cosh \frac{\pi q}{2 \alpha}, \\
\ln \xi(q, T) & =-\ln \alpha+\psi(1)-\operatorname{Re} \psi\left(\frac{1}{2}+\frac{i q}{2 \alpha}\right),
\end{aligned}
$$


where as before $\alpha=2 \pi T$. For our present purposes, the amplitude $A$ is related by eq. (2.15) to the fermions mass $m$, which is considered a parameter independent of $q$ and $T$. So, we view eq. (3.5) as determining the $q$ and $T$ dependence of the amplitude $\widetilde{A}$ in eq. (3.2).

The real part of the digamma function in (3.6) is a monotonically increasing function of $q^{2}$, approaching $\ln (q / \alpha)$ in the limit of large $q / \alpha$. Note, then, that in this limit $\xi$ becomes independent of the temperature, and to logarithmic accuracy $\ln \xi=-\ln q$.

The small-amplitude condition for the scalar, under which the metric deformation is small and eq. (3.2) is applicable, is

$$
\kappa A \ll \xi^{-1},
$$

where $\kappa^{2}=8 \pi G_{N}$, and $G_{N}$ is Newton's constant. Written in terms of the fermion mass in the dual CFT, this becomes

$$
m \ll \xi^{-1},
$$

where we have used the relation $G_{N}=3 /(2 N)$ [15] and eq. (2.15). Since $\xi$ is determined by the larger of $\alpha=2 \pi T$ and $q$, the condition (3.8) is automatically satisfied if $m \ll 2 \pi T$. This is analogous to the Ginzburg-Landau limit in a superconductor.

We now compare eq. (3.1), where we now have an explicit expression for $\ln \xi$, to the order $m^{2}$ correction to the free energy of a multiplet of $N$ free Dirac fermions in two spacetime dimensions. The mass Lagrangian density (in the Lorentzian signature) is

$$
L_{m}=-m\left(e^{-i q x} \bar{\Psi}_{L} \Psi_{R}+e^{i q x} \bar{\Psi}_{R} \Psi_{L}\right),
$$

where $\Psi_{L, R}$ are the left- and right-moving component of the multiplets and $m$ is a positive constant. A chiral transformation will remove the factor $e^{i q x}$ in eq. (3.9), at the price of an additional term appearing in the full Lagrangian:

$$
L=L^{\prime}-\frac{q}{2}\left(\Psi_{R}^{\dagger} \Psi_{R}+\Psi_{L}^{\dagger} \Psi_{L}\right)
$$

where $L^{\prime}$ is the Dirac Lagrangian density with a constant mass $m$. In a superconductor, the quantity in the brackets in (3.10) represents the electric current: recall that we identify the electric charge with the chiral charge of the Dirac fermion and set $v_{F}=1$.

The latter form of the Lagrangian is convenient for finding the spectrum of elementary excitations. There are two branches, with energies

$$
\epsilon_{ \pm}(k)=\sqrt{k^{2}+m^{2}} \pm \frac{1}{2} q \equiv \epsilon_{0}(k) \pm \frac{1}{2} q .
$$

The free energy $\Omega_{F}$ of these fermions is that of the grand canonical ensemble with the chemical potential set to zero. Corrections to $\Omega_{F}$ due to the fermion mass are contained in the first derivative

$$
\frac{\partial \Omega_{F}}{\partial m^{2}}=N \sum_{k} \frac{1}{2 \epsilon_{0}(k)}\left[n_{F}\left(\epsilon_{+}\right)+n_{F}\left(\epsilon_{-}\right)\right]
$$

where $n_{F}(\epsilon)=\left(e^{\beta \epsilon}+1\right)^{-1}$ is the Fermi distribution. Our use of the grand canonical ensemble for an isolated system implies that we are working in the thermodynamic limit, 
when the sum over $k$ can be replaced with an integral. A convenient way to compare the result to the one obtained on the gravity side is to expand both expressions in powers of $q^{2}$ and compare them term by term. The constant, power zero, term in (3.12) is obtained by replacing $\epsilon_{ \pm}$with $\epsilon_{0}$ and is logarithmic:

$$
\frac{N L_{x}}{2 \pi} \int \frac{d k}{\epsilon_{0}(k)} n_{F}\left(\epsilon_{0}\right)=\frac{N L_{x}}{2 \pi}\left(\ln \frac{T}{m}+\text { const }\right) .
$$

This differs from the corresponding term in $\partial \Omega_{\text {grav }} / \partial m^{2}$ by a $T$-independent constant. So, the results for the entropy match. Nonzero powers of $q^{2}$, obtained by expanding eq. (3.12) in $q$, have finite limits at $m=0$. These limits are seen to coincide, term by term, with powers of $q^{2}$ obtained by expanding $\ln \xi$ in $\partial \Omega_{\text {grav }} / \partial m^{2}$.

\section{Entanglement entropy}

The Ryu-Takayanagi (RT) formula [6] for the EE has been argued [7] to follow from a generalized GH formula, combined with a holographic interpretation of the gravitational partition function. In general, one should not expect the RT formula to apply to higherspin gravity; for the case without matter, alternative expressions have been proposed in refs. [16-18]. Given, however, that to the leading order in the mass parameter the higherspin fields do not affect the action, one may wonder if to this order the RT formula remains applicable as well. Here, we compare results obtained from that formula to those from a direct lattice computation in field theory.

For a single interval, application of the RT formula amounts to computing the length of the geodesic, $x(r)$, connecting two given points $x= \pm x_{m}$ on the boundary. As before, the boundary is at a large $r=r_{m}$. The length of the interval is

$$
l=2 x_{m} .
$$

The geodesic has two symmetric branches: one, with $x<0$, going from $r=r_{m}$ to the tip at $r=r_{\text {tip }}$, and the other, with $x>0$, from $r_{\text {tip }}$ back to $r_{m}$. The length of the geodesic in the metric (2.1) is given by

$$
\mathcal{A}[x(r), F(r), G(r)]=2 \int_{r_{\mathrm{tip}}}^{r_{m}}\left[G(r)+F(r)\left(x^{\prime}\right)^{2}\right]^{1 / 2} d r,
$$

where $x^{\prime} \equiv d x / d r$. The geodesic equation for $x(r)$ is obtained by extremizing this at fixed $r_{m}$ and $x_{m}$, with $r_{\text {tip }}$ obtained as a function of these parameters in the course of the procedure. The RT formula for the EE [6], $S_{\text {ent }}=\mathcal{A} /\left(4 G_{N}\right)$, combined with the relation [15] $G_{N}=3 /(2 N)$, then gives

$$
S_{\text {ent }}=\frac{1}{6} N \mathcal{A} .
$$

A simplification specific to $d=2$ is that the integrand in eq. (4.2) is independent of $x$, so the corresponding canonical "momentum" is conserved along the geodesic:

$$
\frac{F(r) x^{\prime}}{\left[G(r)+F(r)\left(x^{\prime}\right)^{2}\right]^{1 / 2}}=\text { const }=\left[F\left(r_{\text {tip }}\right)\right]^{1 / 2} .
$$




\begin{tabular}{|c|ccc|}
\hline$r_{\text {tip }} / \alpha$ & 0.5 & 1 & 2 \\
\hline$l \alpha$ & 2.887 & 1.763 & 0.962 \\
\hline
\end{tabular}

Table 1. The length $l$ of the entangling interval (in units of the thermal wavelength $\alpha^{-1}$, where $\alpha=2 \pi T$ ) as given by the undeformed geodesic (4.7) for cutoff radius $r_{m} / \alpha=10^{3}$ and various values of $r_{\text {tip }}$.

The value of the constant has been found by noting that $x^{\prime} \rightarrow \infty$ at the tip. Moreover, the Hamilton-Jacobi theory applied to the functional (4.2) tells us that the same constant appears as the derivative of $\mathcal{A}$ with respect to the endpoint:

$$
\frac{\partial \mathcal{A}}{\partial x_{m}}=2\left[F\left(r_{\text {tip }}\right)\right]^{1 / 2} .
$$

With the help of eqs. (4.1) and (4.3), this can be expressed as the derivative of the EE with respect to the length of the interval:

$$
\frac{\partial S_{\text {ent }}}{\partial l}=\frac{N}{6}\left[F\left(r_{\text {tip }}\right)\right]^{1 / 2} .
$$

Expression (4.6) is curious in its own right, but is not the most convenient one if we are looking specifically at the case of small deformations (by a scalar of small amplitude $A$ ): it requires us to consider variations of both $F(r)$ and $r_{\text {tip }}$, i.e., of the geodesic itself. In this case, we have found it more convenient to proceed directly from eq. (4.2). Then, to the leading order in the scalar amplitude, we can use the geodesic of the undeformed BTZ background, namely,

$$
x_{0}(r)=\frac{1}{\alpha} \ln \frac{c r+\alpha\left(r^{2}-r_{\mathrm{tip}}^{2}\right)^{1 / 2}}{r_{\mathrm{tip}}\left(r^{2}+\alpha^{2}\right)^{1 / 2}},
$$

where $c \equiv\left(\alpha^{2}+r_{\text {tip }}^{2}\right)^{1 / 2}$ is the value of the constant (4.4) for the undeformed case.

Note that $r_{\text {tip }}$ in eq. (4.7) is the value of $r$ at the tip of the undeformed geodesic. As such, it is different (by terms of order $A^{2}$ ) from $r_{\text {tip }}$ we would have to use in eq. (4.6). On the other hand, in the method employed in what follows, to the required accuracy the two values are interchangeable, so we do not use separate notation for each.

For numerical work, it is convenient to choose $r_{\text {tip }}$ at will and map it by eq. (4.7) to a value of $l=2 x_{m}$. For future use, we present here a table of $\left(r_{\text {tip }}, l\right)$ pairs obtained in this way (table 1 ).

The metric corrections are found from the $x x$ and $r r$ components of the Einstein equations,

$$
\begin{aligned}
& \frac{G^{\prime}}{G}+2 G r=2 \kappa^{2} r\left[-\frac{q^{2} G}{F} \phi^{2}+\left(\phi^{\prime}\right)^{2}+M^{2} G \phi^{2}\right] \\
& \frac{F^{\prime}}{F}-2 G r=2 \kappa^{2} r\left[-\frac{q^{2} G}{F} \phi^{2}+\left(\phi^{\prime}\right)^{2}-M^{2} G \phi^{2}\right]
\end{aligned}
$$

We now use $\phi$ to denote the radial dependence of the scalar and assume it real. The original scalar field is now $e^{i q x} \phi(r)$. Primes denote derivatives with respect to $r$. These equations 


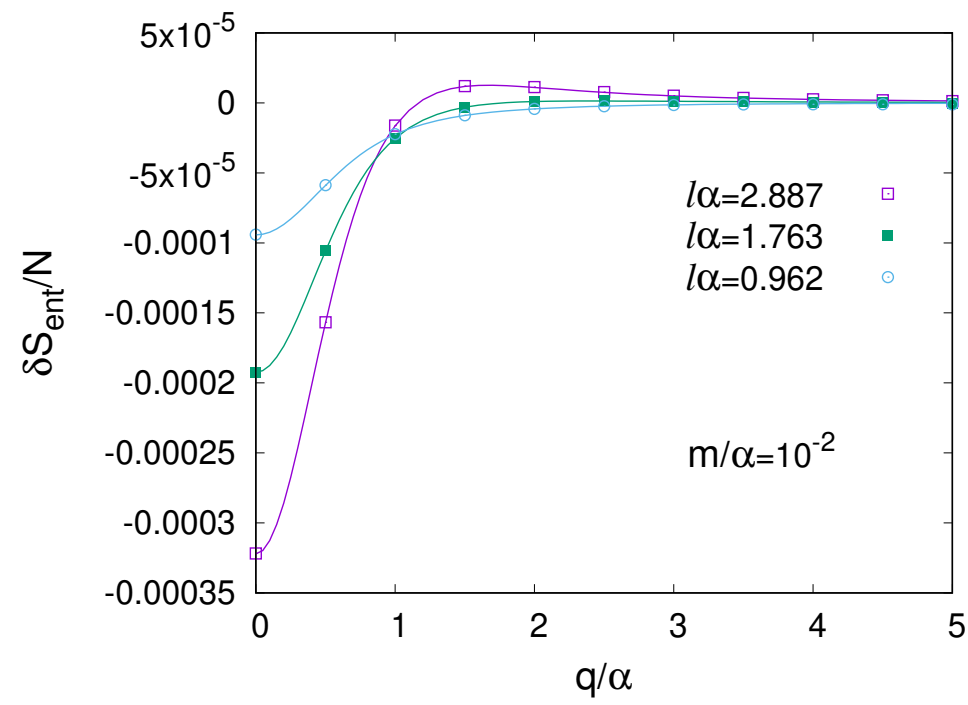

Figure 1. Entanglement entropy change per fermion due to a mass deformation for intervals of different lengths, as a function of the superflow momentum $q$ (in units of $\alpha=2 \pi T$ ). Curves: results of a holographic calculation using general relativity as the dual. Points: results of a direct calculation in lattice field theory. The interval lengths are the same as in table 1.

have to be solved with the boundary conditions

$$
G(0)=1 / \alpha^{2}, \quad F\left(r_{m}\right)=r_{m}^{2},
$$

which correspond to varying the metric with the temperature and length of the $x$ circle fixed. The boundary conditions for $\phi$ can be found from eq. (2.8) (with $\lambda=1$ ), which is applicable since we are working in the linearized theory. We have

$$
\phi(0)=\widetilde{A} / \alpha, \quad \phi^{\prime}(0)=0,
$$

where $\widetilde{A}$ is related to $A$ by eq. (3.5). The system consisting of eqs. (4.8) and (4.9) and the equation for the scalar can now be solved numerically, and the solution can be used together with the geodesic (4.7) in eq. (4.2).

In figure 1 , we plot results obtained by this method for $m / \alpha=10^{-2}, r_{m} / \alpha=10^{3}$, and the values of $r_{\text {tip }}$ shown in table 1 . The length of the geodesic (4.7) in the undeformed geometry is

$$
\mathcal{A}_{0}=2 \ln \left(y+\sqrt{y^{2}-1}\right)
$$

where $y \equiv r_{m} / r_{\text {tip }}$, and we plot the quantity

$$
\frac{\delta S_{\mathrm{ent}}}{N}=\frac{1}{6}\left(\mathcal{A}-\mathcal{A}_{0}\right)
$$

which is the entropy change per fermion, due to a finite mass. This quantity goes to a finite limit at large $r_{m}$.

For comparison, in the same figure, we show results of a direct lattice computation of the EE for the corresponding values of the interval length $l$ (table 1). The method is 
described (for $q=0$ ) in ref. [11]. We use staggered fermions [19] with antiperiodic boundary conditions on a uniform lattice with an even number $N$ of lattice sites. The Hamiltonian corresponding to the additional Lagrangian of eq. (3.10) is discretized as

$$
H_{\mathrm{add}}=\frac{q}{2} \sum_{n=0}^{N-1} \Psi_{n}^{\dagger} \Psi_{n}
$$

where $\Psi_{n}$ is a one-component Fermi operator. The results in figure 1 are for $N=5 \times$ $10^{4}$. The good agreement seen in the figure confirms applicability of general relativity to computation of $O\left(\mathrm{~m}^{2}\right)$ terms in the EE.

Let us comment on short-distance cutoffs required in both calculations. On the gravity side, the cutoff is represented by the maximum radius $r_{m}$ [20]; on the lattice, by the lattice spacing $h$. Because the difference (4.13) is finite in the limit $r_{m} \rightarrow \infty$, the precise relation between $r_{m}$ and $h$ does not matter, as long as $h$ remains much smaller than all the physical length scales. For definiteness, we have set $h=1 / r_{m}$. Then, for instance, for $r_{m} / \alpha=10^{3}$ (the value used for compiling table 1 ), $1 /(h \alpha)=10^{3}$, so $l$ in units of the lattice spacing is obtained by multiplying an entry for $l \alpha$ in table 1 by 1000 .

We have checked that the cutoff of $r_{m} / \alpha=10^{3}$, used in obtaining figure 1 , is already large enough for the results to change little upon increasing it further. The agreement between the results of gravity and lattice calculations remains good. The agreement also extends to larger values of $m / \alpha$, as long as they remain small enough for the $m^{2}$ scaling of $\delta S_{\text {ent }}$ to hold. We have verified that for $m / \alpha=0.03$.

\section{Limit of a short interval}

In the limit of a short interval, $l \ll \xi$, the leading terms in $\delta S_{\text {ent }}$ can be computed analytically. Indeed, in this case, the entire geodesic lies near the boundary, and we can use the asymptotic expression (2.9) for $\phi$ and linearized Einstein equations to find the leading logarithms in the metric functions there. The computation is similar to those done in refs. [21-23] for the vacuum AdS deformed by scalars of different masses. In our case, we need to keep track of deformations of both $G$ and $F$, since both contribute to the geodesic length (4.2). Define the deformations $G_{1}$ and $F_{1}$ by

$$
G(r)=G_{0}(r)+G_{1}(r), \quad F(r)=F_{0}(r)+F_{1}(r) .
$$

In the asymptotic region, linearized eqs. (4.8) and (4.9) become

$$
\begin{aligned}
& \frac{G_{1}^{\prime}}{G_{0}}+4 r G_{1}=\frac{2 \kappa^{2} A^{2}}{r^{3}}[-2 \ln (r \xi)+1+\ldots], \\
& \frac{F_{1}^{\prime}}{F_{0}}-\frac{2 r F_{1}}{F_{0}^{2}}=2 r G_{1}+\frac{2 \kappa^{2} A^{2}}{r^{3}}\left[2 \ln ^{2}(r \xi)-2 \ln (r \xi)+1+\ldots\right] .
\end{aligned}
$$

Here and in the next equation dots denote terms suppressed by inverse powers of $r$. The solution to the first of these is

$$
G_{1}(r)=\frac{2 \kappa^{2} A^{2}}{r^{4}}\left[-\ln ^{2}(r \xi)+\ln r+\text { const }+\ldots\right]
$$


Substituting this into the equation for $F_{1}$, we find that logarithmic terms on the right-hand side all cancel, and the leading behavior of $F_{1}$ at large $r$ is a constant.

To the linear order in $F_{1}$ and $G_{1}$, the change in the length (4.2) is

$$
\delta \mathcal{A}[x(r), F(r), G(r)]=\int_{r_{\mathrm{tip}}}^{r_{m}} \frac{G_{1}(r)+F_{1}(r)\left(x^{\prime}\right)^{2}}{\left[G_{0}(r)+F_{0}(r)\left(x^{\prime}\right)^{2}\right]^{1 / 2}} d r .
$$

As before, we can use here the undeformed geodesic (4.7) since it was extremal to the zeroth order. The term with $F_{1}$ does not produce any logarithms of $r_{\text {tip }}$, so as far as those are concerned

$$
\delta \mathcal{A}[x(r), F(r), G(r)] \approx \int_{r_{\text {tip }}}^{r_{m}}\left(r^{2}-r_{\text {tip }}^{2}\right)^{1 / 2} G_{1}(r) d r .
$$

The integral is convergent in the limit $r_{m} \rightarrow \infty$, and we assume this limit in what follows. Substituting eq. (5.4) for $G_{1}$ and computing the integrals, we obtain

$$
\delta \mathcal{A}[x(r), F(r), G(r)]=\frac{2 \kappa^{2} A^{2}}{3 r_{\text {tip }}^{2}}\left[-\ln ^{2}\left(r_{\text {tip }} \xi\right)+\left(2 \ln 2-\frac{5}{3}\right) \ln r_{\text {tip }}+O(1)\right]
$$

where $O(1)$ refers to counting of powers of $\ln r_{\text {tip }}$. For a near-boundary geodesic, eq. (4.7) can be approximated by

$$
x(r)=\left(\frac{1}{r_{\text {tip }}^{2}}-\frac{1}{r^{2}}\right)^{1 / 2} .
$$

In the limit $r_{m} \rightarrow \infty$, this gives $l=2 x_{m}=2 / r_{\text {tip }}$. Finally, using eq. (2.15) to relate $A$ to the fermion mass, we obtain

$$
\delta S_{\mathrm{ent}}=\frac{1}{6} N m^{2} l^{2}\left[-\ln ^{2}(l / \xi)+\frac{5}{3} \ln l+O(1)\right],
$$

where we now count powers of $\ln l$. The leading dependence of eq. (4.13) on $q$ is due to $\ln \xi$ in

$$
\ln ^{2}(l / \xi)=\ln ^{2} l-2 \ln l \ln \xi+O(1)
$$

with $\ln \xi$ given by eq. (3.6). Note that, in the limit $q \gg T$, expression (5.10) becomes independent of the temperature.

Our results so far have been for the case $m \ll 1 / \xi$, where $\xi$ is determined by the larger of $T$ and $q$. Let us comment on the counterpart of eq. (5.9) for the opposite case, when $m$ is the largest mass scale in the problem. For example, let us consider deformations of the global $\mathrm{AdS}_{3}$ by a scalar of the form (2.8) subject to the condition $|q| \ll m$. This is appropriate for the system at zero temperature and a low superflow speed. The metric is

$$
d s_{T=0}^{2}=F(r) d \tau^{2}+r^{2} d x^{2}+G(r) d r^{2} .
$$

The undeformed AdS of unit radius corresponds to $F(r)=[G(r)]^{-1}=1+r^{2}$. The dual CFT now lives on a unit circle: $L_{x}=2 \pi$. As before, we focus on a scalar of mass $M^{2}=-1$ corresponding to the operator $O=\bar{\Psi}_{R} \Psi_{L}$ in the free-fermion CFT. The amplitude $A$ is related to the mass $m$ of the fermion by the same eq. (2.15). The key difference from the 
preceding case is that the correlation length in the CFT is now determined by the mass, and the role of the thermodynamic limit is taken over by the condition

$$
m \gg 2 \pi / L_{x}=1 .
$$

For this condition to apply, the amplitude of the scalar must be relatively large, and as result the metric deformation cannot be small everywhere. Indeed, we can define the correlation length holographically as the radius $r$ of the region where the metric correction is comparable to the unperturbed metric.

As the scalar decreases at large $r$, eventually, at $r \gg 1 / \xi$, the metric deformation becomes small. We are back in the domain of linearized theory, where the asymptotic formula (2.9) for the scalar applies. The correlation length $\xi$ in it, however, is now determined primarily by the mass, and the linearized theory provides no information on it beyond the estimate $\xi \sim m^{-1}$. Working in parallel with the computation above, we obtain the same eq. (5.9) for short intervals; however, uncertainty in the estimate $\xi \sim m^{-1}$ prevents one from determining the coefficient of the $\ln l$ term. The result is

$$
\delta S_{\mathrm{ent}}=\frac{1}{6} N m^{2} l^{2}\left[-\ln ^{2}(m l)+O(\ln l)\right],
$$

for $m l \ll 1$. We note that the leading correction in this case coincides with that obtained in ref. [11] by a direct calculation in field theory. Agreement up to a numerical factor, between the $\ln ^{2} l$ terms in the holographic and field-theory calculations for $\Delta=1$, has been noted in ref. [23]. Here, we show that the coefficients match as well.

\section{Discussion}

In this paper, we have aimed to understand conditions under which the entropies of the $d=2$ free-fermion CFT deformed by an $x$-dependent mass term $m e^{i q x}$ can be computed holographically using standard general relativity as the dual. We have considered both the thermal entropy (of the entire space) and the entanglement entropy (EE) of an interval. In both instances, we have found that one can use general relativity to compute the entropy to order $m^{2} \xi^{2}$, where $\xi$ is the correlation length set by the larger of the temperature and the momentum $q$. For the thermal entropy, this can be seen as a consequence of the proposed duality [8] between this CFT and the higher-spin theory of ref. [9]: to order $m^{2}$, the effective actions in that theory and in general relativity are the same.

For the EE, the reasoning is less direct since in that case, before applying duality, one makes transformations of the CFT coordinates and metric $[5,7]$. One could argue, however, that, while these transformations can make the coordinate dependence of the fermion mass complicated, they do not affect counting of the powers of $m$, so to compute the entropy to order $m^{2}$ one may still be able to replace the higher-spin gravity dual with the standard one. Our results lend support to this argument.

Finally, we remark that holographic formulas for the EE in higher-spin $\mathrm{AdS}_{3}$ gravity without matter have been proposed (within the Chern-Simons formulation) in refs. [16-18]. One may expect that, in the presence of a scalar necessary to describe a mass deformation, these formulas will have to be modified. It would be interesting to find out how. 


\section{Acknowledgments}

We thank M. Kruczenski for discussions. This work was supported in part by the DOE QuantISED program (through Grant No. DE-SC0019202 and the consortium "Intersections of QIS and Theoretical Particle Physics" at Fermilab) and by the W.M. Keck Foundation.

Open Access. This article is distributed under the terms of the Creative Commons Attribution License (CC-BY 4.0), which permits any use, distribution and reproduction in any medium, provided the original author(s) and source are credited.

\section{References}

[1] G.W. Gibbons and S.W. Hawking, Action Integrals and Partition Functions in Quantum Gravity, Phys. Rev. D 15 (1977) 2752 [InSPIRE].

[2] J.M. Maldacena, The Large $N$ limit of superconformal field theories and supergravity, Int. J. Theor. Phys. 38 (1999) 1113 [Adv. Theor. Math. Phys. 2 (1998) 231] [hep-th/9711200] [INSPIRE].

[3] S.S. Gubser, I.R. Klebanov and A.M. Polyakov, Gauge theory correlators from noncritical string theory, Phys. Lett. B 428 (1998) 105 [hep-th/9802109] [INSPIRE].

[4] E. Witten, Anti-de Sitter space and holography, Adv. Theor. Math. Phys. 2 (1998) 253 [hep-th/9802150] [INSPIRE].

[5] H. Casini, M. Huerta and R.C. Myers, Towards a derivation of holographic entanglement entropy, JHEP 05 (2011) 036 [arXiv:1102.0440] [INSPIRE].

[6] S. Ryu and T. Takayanagi, Holographic derivation of entanglement entropy from AdS/CFT, Phys. Rev. Lett. 96 (2006) 181602 [hep-th/0603001] [InSPIRE].

[7] A. Lewkowycz and J. Maldacena, Generalized gravitational entropy, JHEP 08 (2013) 090 [arXiv: 1304.4926] [INSPIRE].

[8] M.R. Gaberdiel and R. Gopakumar, An AdS 3 Dual for Minimal Model CFTs, Phys. Rev. D 83 (2011) 066007 [arXiv:1011.2986] [INSPIRE].

[9] S.F. Prokushkin and M.A. Vasiliev, Higher spin gauge interactions for massive matter fields in 3 -D AdS space-time, Nucl. Phys. B 545 (1999) 385 [hep-th/9806236] [INSPIRE].

[10] M. Bañados, C. Teitelboim and J. Zanelli, The Black hole in three-dimensional space-time, Phys. Rev. Lett. 69 (1992) 1849 [hep-th/9204099] [INSPIRE].

[11] H. Casini, C.D. Fosco and M. Huerta, Entanglement and alpha entropies for a massive Dirac field in two dimensions, J. Stat. Mech. 0507 (2005) P07007 [cond-mat/0505563] [INSPIRE].

[12] I.R. Klebanov and E. Witten, AdS/CFT correspondence and symmetry breaking, Nucl. Phys. B 556 (1999) 89 [hep-th/9905104] [INSPIRE].

[13] M. Tinkham, Introduction to Superconductivity, 2nd edition, Mineola, NY, Dover Publications (2004).

[14] M. Abramowitz and I.A. Stegun, Handbook of Mathematical Functions, 10th printing, Washington, DC, National Bureau of Standards (1972). 
[15] J.D. Brown and M. Henneaux, Central Charges in the Canonical Realization of Asymptotic Symmetries: An Example from Three-Dimensional Gravity, Commun. Math. Phys. 104 (1986) 207 [INSPIRE].

[16] M. Ammon, A. Castro and N. Iqbal, Wilson Lines and Entanglement Entropy in Higher Spin Gravity, JHEP 10 (2013) 110 [arXiv:1306.4338] [INSPIRE].

[17] J. de Boer and J.I. Jottar, Entanglement Entropy and Higher Spin Holography in AdS $S_{3}$, JHEP 04 (2014) 089 [arXiv: 1306.4347] [INSPIRE].

[18] A. Castro and E. Llabrés, Unravelling Holographic Entanglement Entropy in Higher Spin Theories, JHEP 03 (2015) 124 [arXiv: 1410.2870] [INSPIRE].

[19] L. Susskind, Lattice Fermions, Phys. Rev. D 16 (1977) 3031 [InSPIRE].

[20] L. Susskind and E. Witten, The Holographic bound in anti-de Sitter space, hep-th/9805114 [INSPIRE].

[21] H. Liu and M. Mezei, A Refinement of entanglement entropy and the number of degrees of freedom, JHEP 04 (2013) 162 [arXiv: 1202.2070] [INSPIRE].

[22] I.R. Klebanov, T. Nishioka, S.S. Pufu and B.R. Safdi, On Shape Dependence and RG Flow of Entanglement Entropy, JHEP 07 (2012) 001 [arXiv:1204.4160] [INSPIRE].

[23] T. Nishioka, Relevant Perturbation of Entanglement Entropy and Stationarity, Phys. Rev. D 90 (2014) 045006 [arXiv:1405.3650] [INSPIRE]. 